\title{
Continuous wavelet transform ridge extraction for spectral interferometry imaging
}

Michael Liebling, Thierry-Francois Bernhard, Adrian $\mathrm{H}$. Bachmann, Luc Froehly, Theo Lasser, et al.

Michael Liebling, Thierry-Francois Bernhard, Adrian H. Bachmann, Luc Froehly, Theo Lasser, Michael Unser, "Continuous wavelet transform ridge extraction for spectral interferometry imaging," Proc. SPIE 5690, Coherence Domain Optical Methods and Optical Coherence Tomography in Biomedicine IX, (13 April 2005); doi: 10.1117/12.591440

SPIE. Event: SPIE BiOS, 2005, San Jose, CA, United States 


\title{
Continuous Wavelet Transform Ridge Extraction for Spectral Interferometry Imaging
}

\author{
Michael Liebling ${ }^{1}$, Thierry-François Bernhard ${ }^{2}$, Adrian H. Bachmann², \\ Luc Froehly ${ }^{2}$, Theo Lasser ${ }^{2}$, Michael Unser ${ }^{2}$ \\ ${ }^{I}$ Biological Imaging Center, Beckman Institute, California Institute of Technology, \\ Mail Code 139-74, Pasadena, CA 91125, USA; \\ ${ }^{2}$ Institute of Imaging and Applied Optics, Swiss Federal Institute of Technology Lausanne, \\ Bâtiment de Microtechnique 4.127, CH-1015 Lausanne, Switzerland
}

\begin{abstract}
The combination of wavelength multiplexing and spectral interferometry allows for the encoding of multidimensional information and its transmission over a mono-dimensional channel; for example, measurements of a surface's topography acquired through a monomode fiber in a small endoscope. The local depth of the imaged object is encoded in the local spatial frequency of the signal measured at the output of the fiber-decoder system. We propose a procedure to retrieve the depth-map by determining the signal's instantaneous frequency. First, we compute its continuous, complex-valued, wavelet transform (CWT). The frequency signature at every position is contained in the resulting scalogram. We then extract the ridge of maximal response by use of a dynamic programming algorithm thus directly recovering the object's topography. We present results that validate this procedure based on both simulated and experimental data.
\end{abstract}

Keywords: Continuous wavelet transform, dynamic programming, ridge extraction, spectral interferometry, wavelength multiplexing, temporal holography, optical coherence tomography

\section{INTRODUCTION}

Recently, a new setup for imaging surface topography based on the encoding and transmission of the local depth information by means of wavelength multiplexing ${ }^{1,2}$ and spectral interferometry ${ }^{3}$ was proposed. ${ }^{4}$ Because the local depth information is encoded such that it may be transmitted over a mono-dimensional channel (e.g. a monomode fiber) this method is of highest interest for endoscopy. Indeed, accurate depth measurement is crucial for both diagnostic and treatment of tracheal and bronchial stenoses. In this paper, we propose a new method for retrieving the sample's local depth by digitally processing the decoded signal.

We use the following definition of the Fourier transform $\hat{f}(\nu)$ of a function $f(x)$

$$
\begin{aligned}
\hat{f}(\nu) & =\mathcal{F} f(\nu)=\int_{-\infty}^{\infty} f(x) \exp (-2 i \pi t \nu) \mathrm{d} t, \\
f(t) & =\mathcal{F}^{-1} \hat{f}(t)=\int_{-\infty}^{\infty} \hat{f}(\nu) \exp (2 i \pi \nu t) \mathrm{d} \nu .
\end{aligned}
$$

With this definition, $\|f\|=\|\hat{f}\|$.

\section{SETUP AND MODELLING}

The experimental setup, depicted in Fig 1, can be modeled as sketched in the block diagram of Fig. 2. A pulse $p(t)$ emitted from a white light femtosecond source is sent through the fiber, expanded, and reaches a grating. Two lenses map the \pm 1 diffraction orders onto the sample and a reference mirror, respectively. The combination of the grating-lens system can be modeled by a temporal Fourier transform that maps each frequency (or wavelength) of the pulse to a specific spot on the sample. The sample's local depth and reflectivity introduce a phase shift in the signal at the corresponding position (or equivalently wavelength). The object (and reference) signals follow a reverse path into the fiber. The process can be approximated by a filtering operation with a filter $h(x)=a(x) \exp (i \phi(x))$. The recombined signal is transmitted over the fiber and decoded by another grating-lens system and finally detected (square-law detection).

M.L.: E-mail: liebling@ caltech.edu, Telephone: (+1-626) 395-2863 


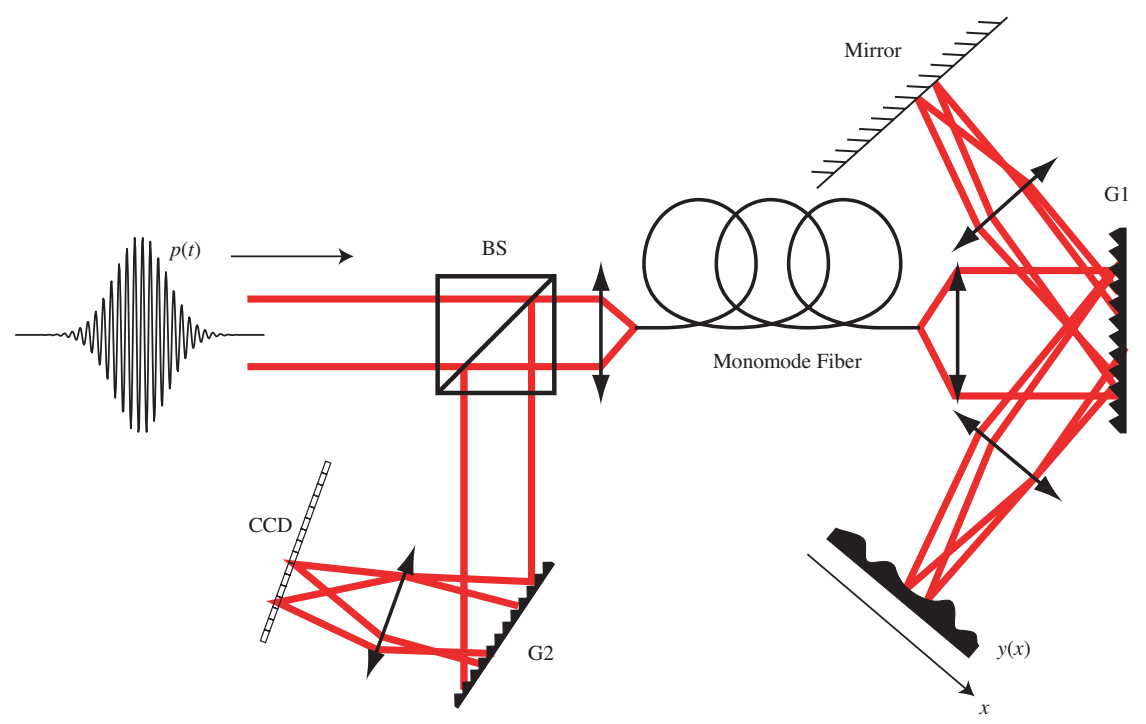

Figure 1. Optical setup for wavelength multiplexing spectral interferometry imaging. BS: beam-splitter, G1, G2: gratings.

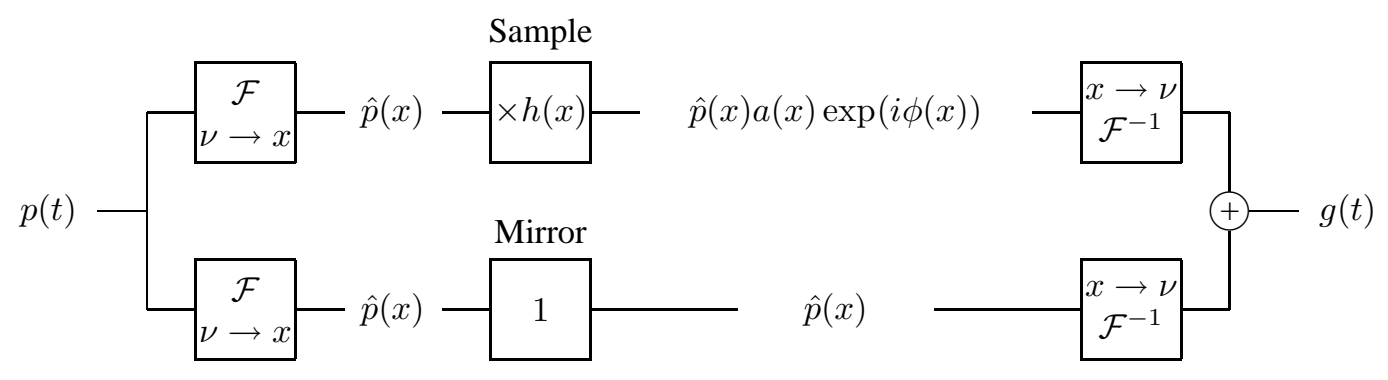

(a)

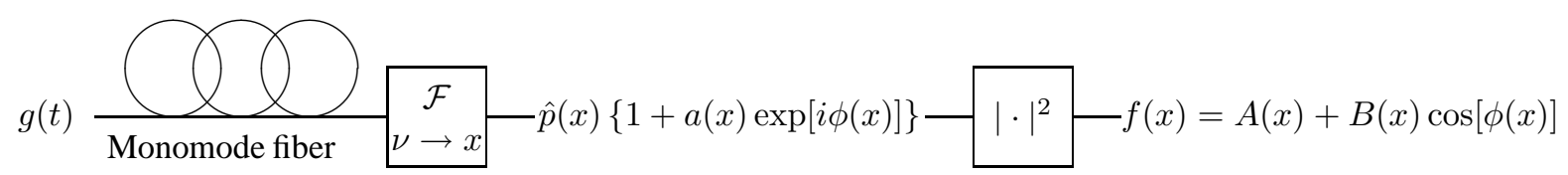

(b)

Figure 2. Block diagrams of (a) Acquisition and encoding (b) Transmission and decoding.

\section{ALGORITHM}

\subsection{Measured Signal}

The interference pattern measured on the CCD is of the form

$$
f(x)=A(x)+B(x) \cos [\phi(x)] .
$$

The signal $f(x)$ has a local phase $\phi(x)$ that is directly related to the local depth of the sample and a slow varying envelope $B(x)$ and DC component $A(x)$. In order to retrieve $\phi(x)$, we take advantage of the continuous wavelet transform formalism. ${ }^{5,6}$ The latter has proven to be effective for many time (or space)-frequency applications, including some in optics. $^{7-9}$ 


\subsection{Continuous Wavelet Analysis}

A wavelet is a function $\psi \in L^{2}(\mathbb{R})$ with zero average

$$
\int_{-\infty}^{\infty} \psi(x) \mathrm{d} x=0
$$

It is normalized such that $\|\psi\|=1$ and centered in the neighborhood of $x=0$. A family of time-frequency atoms is obtained by scaling $\psi$ by $s$ and translating it by $u$ :

$$
\psi_{u, s}(x)=\frac{1}{\sqrt{s}} \psi\left(\frac{x-u}{s}\right)
$$

with $\left\|\psi_{u, s}\right\|=1$. The wavelet transform of $f \in L^{2}(\mathbb{R})$ at position $u$ and scale $s$ is

$$
\mathcal{W}_{f}(u, s)=\left\langle f, \psi_{u, s}\right\rangle=\int_{-\infty}^{\infty} f(t) \frac{1}{\sqrt{s}} \psi^{*}\left(\frac{x-u}{s}\right) \mathrm{d} x .
$$

We construct an analytical (complex-valued) wavelet by modulating a Gaussian window

$$
\psi(x)=\left(\frac{2}{\pi \sigma^{2}}\right)^{1 / 4} \exp \left(2 i \pi \nu_{0} x\right) \exp \left(-x^{2} / \sigma^{2}\right) .
$$

Its Fourier transform,

$$
\hat{\psi}(\nu)=\left(2 \pi \sigma^{2}\right)^{1 / 4} \exp \left[-\pi^{2} \sigma^{2}\left(\nu-\nu_{0}\right)^{2}\right]
$$

is analytical for $\nu_{0} \gg 0$.

The CWT of a real signal using an analytic wavelet has the property that the derivative of a point on the ridge vanishes

$$
\frac{\partial \Psi_{f}}{\partial s}(u, s)=0, \quad \Psi_{f}(u, s)=\arg \left(\mathcal{W}_{f}(u, s)\right)
$$

We take advantage of this (sufficient) condition to extract the ridge.

\subsection{Ridge Extraction via Dynamic Programming}

After the signal's CWT has been calculated, the problem of finding the depth at every $x$ is isomorphic to that of finding a ridge of maximum response through the scalogram. We address this problem by using a dynamic programming algorithm. ${ }^{10}$ It was shown that algorithms based on that technique are highly effective for that purpose. ${ }^{11}$ One such method has been utilized in the context of a CWT-based Moiré imaging technique. ${ }^{12}$ Here, to improve accuracy, we rely on two criteria to ensure that the right frequency is chosen. We compute a new image based on the absolute value and phase of the signal's CWT:

$$
i(x, y)=\left|\mathcal{W}_{f}(x, y)\right| \gamma\left(\frac{\mathrm{d} \Psi_{f}(u, s)(x, y)}{\mathrm{d} y}\right),
$$

where $\gamma(u)$ is a weighting function that penalizes pixels that do not satisfy the ridge condition (9)

$$
\gamma(u)= \begin{cases}1-u / u_{m} & \text { if }|u|<u_{m} \\ 0 & \text { otherwise }\end{cases}
$$

with $u_{m}$ a user specified threshold.

Based on the combined information $i(x, y) \in \mathbb{R}$ of the CWT's amplitude $\left|\mathcal{W}_{f}(x, y)\right|$ and phase $\arg \left(\mathcal{W}_{f}(x, y)\right)$, we proceed in three steps that conduct to the extraction of the ridge $y(x)$ (see Fig. 3). 


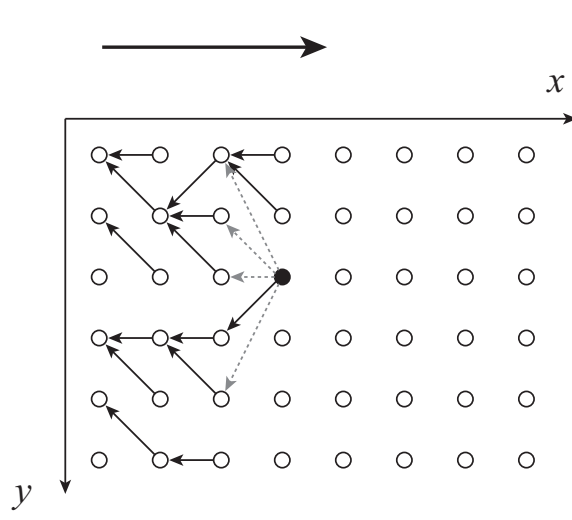

(a)

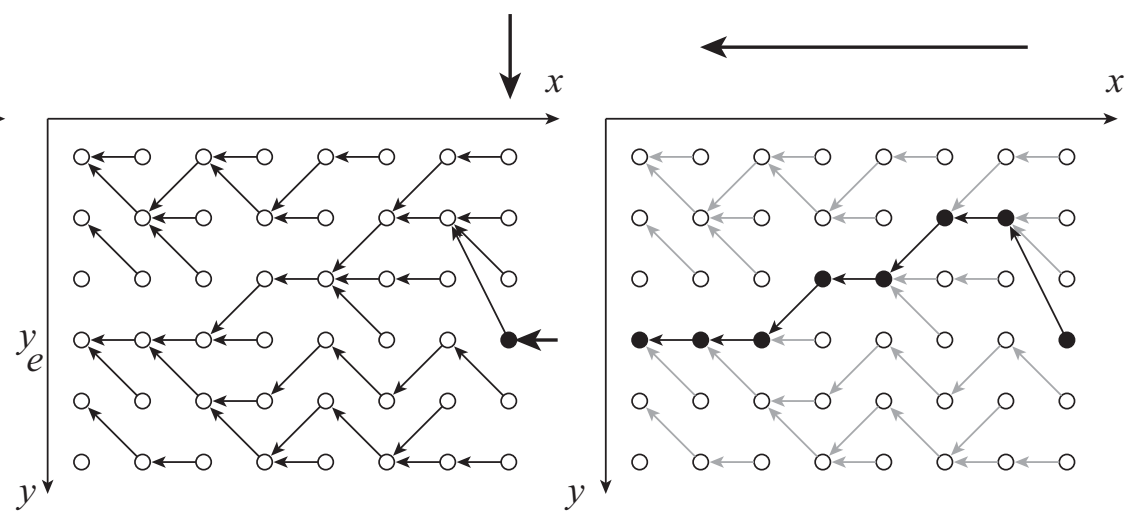

(b)

(c)

Figure 3. The three steps of the dynamic programming ridge extraction procedure.

For $x=0, \ldots, N_{x}-1$, we compute a merit function $Q\left(x, y, y_{p}\right)$ and store the position of the predecessor pixel $\bar{y}_{p}(x, y)$ that yielded the maximal value of the merit function (see Fig. 3(a)), viz.

$$
\left.\begin{array}{rl}
Q\left(x, y, y_{p}\right) & = \begin{cases}Q\left(x-1, y_{p}\right)+i(x, y)-\mu \sigma\left(y, y_{p}\right) & \text { if } x>0 \\
i(x, y) & \text { if } x=0\end{cases} \\
\bar{Q}(x, y) & =\underset{y_{p} \in\left\{y_{\sin }, \ldots, y_{\max }\right\}}{\max } Q\left(x, y, y_{p}\right), \quad x=0, \ldots, N_{x}-1
\end{array}\right\}
$$

$y_{\min }=\max (y-w, 0)$ and $y_{\max }=\min \left(y+w, N_{y}-1\right)$. The function $\sigma\left(y, y_{p}\right)=\left\|y-y_{p}\right\|$, weighted with a factor $\mu>0$, penalizes large leaps. With high probability, the maximum in Eqs. (13) and (14) is unique. When $\bar{Q}(x, y)$ and $\bar{y}_{p}(x, y)$ have been calculated for all $(x, y)$, the abscissa $y_{e}$ (initialization, see Fig. 3(b)) is chosen such that

$$
y_{e}=\arg \max _{y \in\left\{0, \ldots, N_{y}-1\right\}} \bar{Q}\left(N_{x}-1, y\right) .
$$

The curve that maximizes the criterion is then recovered recursively (from right to left, see Fig. 3(c)), i.e.

$$
y(x)=\bar{y}_{p}(x+1, y(x+1)), \quad x=N_{x}-2, \ldots, 0 .
$$

\subsection{Computational complexity}

The computational complexity of the CWT algorithms, that uses an FFT internally, is $\mathcal{O}(N \log N)$, where $N$ is the number of considered signal samples. The dynamic programming algorithm has a linear complexity in the number of computed wavelet coefficients.

\section{RESULTS}

In Fig. 4, we show the different steps that lead from the output signal $f(t)$ measured on the CCD to the extracted ridge $y(x)$. We have evaluated the procedure on synthetic (Fig. 4(a)) and experimental (Fig. 4(b)) data. For the synthetic data, the agreement between the gold standard step function and the recovered ridge is excellent (see bottom of Fig. 4(a)). Owing to the fact that the wavelet transform is local, the technique is robust and not affected by local defects in the measured signal. In Fig. 4(b), the sample—an inclined plane—is well reproduced by the ridge as well. 

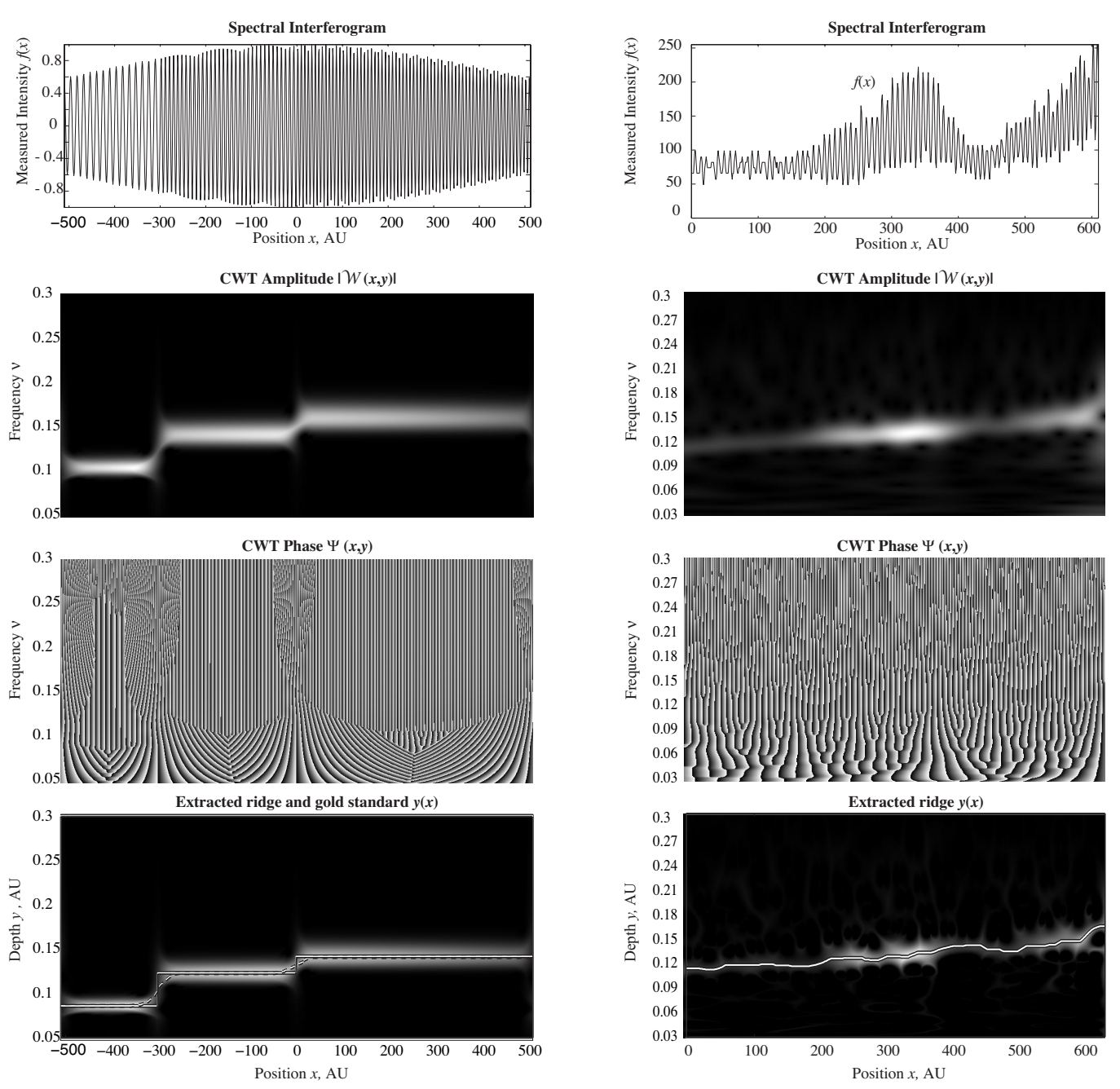

(a)

(b)

Figure 4. (a) Simulated data set. (b) Experimental data set.

\section{CONCLUSION}

We have proposed a CWT and ridge extraction algorithm to retrieve the local depth from spectral interferometry data. Using both simulated and experimental data, we have shown that this technique is suitable for this imaging application. Moreover, the method is suitable for the decoding of several frequencies at a single position, which is not possible using other methods that rely solely on frequency analysis, ${ }^{13}$ and should permit its future extension to extract several interfaces in depth.

\section{ACKNOWLEDGMENTS}

M. Liebling is supported by a fellowship from the Swiss National Science Foundation, PBEL2-104418.

\section{REFERENCES}

1. H. Bartelt, "Wavelength multiplexing for information- transmission," Opt. Comm. 27(3), pp. 365-368, 1978.

2. A. Lacourt and P. Boni, "Transmission of images and holograms using optical fiber tape by means of chromatic encoding," Opt. Comm. 27(1), pp. 57-60, 1978. 
3. C. Froehly, A. Lacourt, and J. C. Viénot, "Time impulse response and time frequency response of optical pupils. Experimental confirmations and applications," Nouvelle Revue d'Optique 4, pp. 183-196, July 1973.

4. L. Froehly, S. Nieto Martin, T. Lasser, C. Depeursinge, and F. Lang, "Multiplexed 3D imaging using wavelength encoded spectral interferometry: a proof of principle," Opt. Commun. 222, pp. 127-136, Aug. 2003.

5. A. Grossmann and J. Morlet, "Decomposition of Hardy functions into square integrable wavelets of constant shape," SIAM Journal on Mathematical Analysis 15(4), pp. 723-736, 1984.

6. N. Delprat, B. Escudié, P. Guillemain, R. Kronland-Martinet, P. Tchamitchian, and B. Torrésani, "Asymptotic wavelet and Gabor analysis-Extraction of instantaneous frequencies," IEEE Trans. Inf. Theory 38, pp. 644-664, Mar. 1992.

7. D. Mendlovic, J. Garcia, Z. Zalevsky, E. Marom, D. Mas, C. Ferreira, and A. W. Lehmann, "Wavelength-multiplexing system for single-mode image transmission," Appl. Opt. 36, pp. 8474-8480, Nov. 1997.

8. J. J. Esteve-Taboada, T. García, C. Ferreira, D. Mendlovic, and Z. Zalevsky, "Two-dimensional optical wavelet decomposition with white-light illumination by wavelength multiplexing," J. Opt. Soc. Am. A 18, pp. 157-163, Jan. 2001.

9. A. Federico and G. H. Kaufmann, "Evaluation of the continuous wavelet transform method for the phase measurement of electronic speckle pattern interferometry fringes," Opt. Eng. 41, pp. 3209-3216, Dec. 2002.

10. R. Bellman, Dynamic Programming, Princeton Univ. Press, Princeton NJ, USA, 1957.

11. M. Unser, G. Pelle, P. Brun, and M. Eden, "Automated extraction of serial myocardial borders from M-mode echocardiograms," IEEE Trans. Medical Imag. 8, pp. 96-103, March 1989.

12. H. Liu, A. N. Cartwright, and C. Basaran, "Moiré interferogram phase extraction: a ridge detection algorithm for continuous wavelet transforms," Appl. Opt. 43, pp. 850-857, Feb. 2004.

13. S. L. Marple, "Computing the discrete-time "analytic" signal via FFT," IEEE Transactions on Signal Processing 47, pp. 2600-2603, Sept. 1999. 